\title{
Qualidade de vida em idosos que sofreram quedas: revisão integrativa da literatura
}

\author{
Quality of life in elderly people that have suffered falls: \\ integrative literature review
}

\author{
Adriana Cristina Nicolussi ${ }^{1}$ \\ Jack Roberto Silva Fhon ${ }^{1}$ \\ Claudia Aline Valente Santos ${ }^{1}$ \\ Luciana Kusumota ${ }^{1}$ \\ Sueli Marques ${ }^{1}$ \\ Rosalina Aparecida Partezani Rodrigues ${ }^{1}$
}

${ }^{1}$ Escola de Enfermagem de Ribeirão Preto, Universidade de São Paulo (USP). Av. Bandeirantes 3900, Campus

Universitário. 14040-902

Ribeirão Preto SP.

drinicolussi@usp.br

\begin{abstract}
An Integrative Literature Review was conducted in order to evaluate the scientific knowledge produced regarding quality of life of the elderly who have suffered falls. Published articles between 1999 and 2009 were selected from the LILACS, CINAHL and MEDLINE databases, and the following search words in Portuguese, English and Spanish were used: quality of life, the elderly and accidents involving falls. Nine articles met the inclusion criteria. It was revealed that falls are frequent among the elderly, and especially the elderly with more intrinsic risk factors and who have suffered falls are those who are more afraid and more prone to fall again. They also reported physical, mental and emotional impairments, body pains and environmental problems. It was revealed that programs to prevent falling can improve the quality of life of the elderly over time. It was clear that there are knowledge gaps, especially a low level of national scientific output, especially by nurses and the predominance of descriptive studies, with low levels of data. We suggest that health professionals conduct research into interventions to prevent falls that can be used in clinical practice in improving the quality of life of the elderly.
\end{abstract}

Key words Quality of life, Elderly, Accidental falls, Health of the elderly, Review
Resumo Foi realizada uma Revisão Integrativa da Literatura com o objetivo de avaliar o conhecimento científico produzido relacionado à qualidade de vida do idoso que sofreu quedas. Métodos: foram selecionados artigos publicados nas bases de dados Lilacs, CINAHL e Medline, com os seguintes descritores: qualidade de vida, idoso e acidentes por quedas, nos idiomas português, inglês e espanhol, entre 1999 e 2009. Resultados: nove artigos atenderam aos critérios de inclusão. Encontrou-se que as quedas são frequentes nos idosos, sendo que aqueles com mais fatores de risco intrínsecos e que já caíram apresentaram mais medo e possuem mais chance de cairem novamente; e também relataram déficits nas funções física, mental/emocional, dor corporal e relacionados ao meio ambiente e que programas de prevenção de quedas podem melhorar sua qualidade de vida com o tempo. Conclusão: evidenciou-se como lacunas no conhecimento: escassa produção científica nacional, principalmente de autores enfermeiros, e predominio de estudos descritivos e com nivel de evidência considerado fraco. Sugere-se a atuação dos profissionais de saúde em pesquisas de intervenções para prevenção de quedas as quais possam ser aplicadas na prática clínica e que possibilitem melhorar a qualidade de vida dos idosos.

Palavras-chave Qualidade de vida, Idoso, Acidentes por quedas, Saúde do idoso, Revisão 


\section{Introdução}

O crescente aumento da população idosa em todo o mundo, demonstrado nos estudos demográficos e epidemiológicos, evidencia para os órgãos governamentais e para a sociedade constantes desafios, principalmente, no que se refere à área da saúde e aos aspectos socioeconômicos, próprios do envelhecimento populacional. Há cerca de quatro décadas este crescimento da população idosa tem sido observado particularmente nos países em desenvolvimento. No Brasil, este fenômeno revela um crescimento exponencial, cuja projeção, para o ano de 2025, mostra que o número de indivíduos com idade igual ou superior a 60 anos será de 32 milhões ${ }^{1-2}$.

Em decorrência de tal quadro, a queda, considerada um evento não intencional cujo resultado é a mudança de posição do indivíduo para um nível mais baixo em relação a sua posição inicial $^{3}$, tem se tornado uma ocorrência frequente e um problema crescente com o processo de envelhecimento. Quanto mais frágil o idoso, maior a propensão à queda, caracterizando um fator importantíssimo de morbidade, institucionalização e mortalidade.

O risco de cair aumenta significativamente com o avançar da idade. A previsão é de que um terço dos idosos que vivem na comunidade cairá no prazo de um ano e, entre os institucionalizados, esta previsão aumenta para 50\%. Ao cair, cerca de 5\% dos idosos necessitam de hospitalização, principalmente por fratura de quadril, e, em cada três casos, um dos pacientes falece no prazo de um ano ${ }^{4}$.

O passo fundamental para a prevenção das quedas é o reconhecimento e a correção dos fatores de risco envolvidos na sua ocorrência, e estes se dividem em intrínsecos e extrínsecos. Os fatores intrínsecos estão relacionados com as alterações fisiológicas do processo de envelhecimento, tais como: idade, presença de múltiplas doenças crônicas, polifarmácia, depressão, diminuição da cognição, redução da capacidade funcional, entre outras. Enquanto os fatores extrínsecos estão relacionados com o meio ambiente e, também, possuem papel importante nos episódios de quedas ${ }^{5-6}$.

As quedas podem acarretar fraturas e medo constante de cair, limitando progressivamente a participação dos idosos em atividades cotidianas. Quedas frequentes em idosos estão associadas à elevada morbidade e mortalidade ${ }^{7}$ e, como consequências comuns, observam-se os prejuízos nas capacidades funcionais e cognitivas, a institucionalização e o aumento de gastos para os serviços de saúde e sociais ${ }^{8}$.

Na perspectiva da saúde pública, há uma atenção crescente relacionada ao impacto das quedas e às morbidades associadas, em decorrência do aumento dos custos com os cuidados à saúde bem como a melhora da Qualidade de Vida (QV) dos idosos ${ }^{9}$. A medida da QV é importante para a avaliação dos efeitos do tratamento e do atendimento pelos serviços de saúde no bem-estar dos clientes. Medidas de QV foram desenvolvidas para entender os tipos de impacto que as intervenções de cuidados à saúde têm na vida dos clientes e o custo-efetividade das mesmas comparadas com outras intervenções de cuidados à saúde ${ }^{10}$.

Para a elaboração de políticas, ações e intervenções de cuidados à saúde que visem melhorar a QV do idoso, é preciso compreender este termo e averiguar qual é o seu significado para esta faixa etária.

Na saúde, existem duas vertentes quanto à conceituação de QV, uma genérica, elaborada pela Organização Mundial de Saúde, na qual a "Qualidade de Vida é a percepção do indivíduo sobre a sua posição na vida, no contexto da cultura e dos sistemas de valores nos quais ele vive, e em relação a seus objetivos, expectativas, padrões e preocupações" ${ }^{\prime 1}$, e a outra, amplamente usada na literatura, estabelece relações mais diretas com enfermidades ou intervenções em saúde, a Qualidade de Vida Relacionada à Saúde (QVRS) que pode ser definida como um ótimo nível de função mental, física, social e de papel desempenhado na vida, abrangendo relacionamentos, percepção de saúde, aptidões, nível de satisfação com a vida e sensação de bem-estar, e também relaciona as perspectivas futuras e a satisfação do paciente com seu tratamento, seus resultados e seu estado de saúde ${ }^{12}$.

Quanto ao conhecimento dos idosos sobre QV, um estudo ${ }^{13}$ realizado com três grupos revelou que o primeiro a conceituou fazendo associações a relacionamentos interpessoais, ao equilíbrio emocional e à boa saúde; o segundo a associou aos hábitos saudáveis, ao lazer e aos bens materiais; e o terceiro a relacionou à espiritualidade, ao trabalho, à retidão e à caridade, ao conhecimento e aos ambientes favoráveis; ou seja, a QV está relacionada à incorporação de sua vida aos seus ideais.

Compreendendo a importância das consequências das quedas para os idosos, os conceitos de QV e a necessidade de implementar políticas e planejar ações de atenção ao idoso, este estudo se 
propõe a avaliar o conhecimento científico produzido, relacionado às quedas e à $\mathrm{QV}$ em idosos. $\mathrm{A}$ questão norteadora foi: "Qual é o conhecimento científico produzido que está relacionado à qualidade de vida dos idosos que sofreram quedas?"

Nesta perspectiva, considerando que os idosos podem apresentar a QV influenciada pelas quedas, o presente estudo teve como objetivo geral buscar e avaliar as evidências disponíveis na literatura sobre o conhecimento científico produzido relacionado à $\mathrm{QV}$ do idoso que sofreu queda. E, como objetivos específicos: caracterizar a produção científica segundo a metodologia adota$\mathrm{da}$, os aspectos relacionados à $\mathrm{QV}$ apresentados pelo idoso que sofreu queda e identificar quais são os instrumentos utilizados para avaliar a QV.

\section{Métodos}

Utilizou-se a Revisão Integrativa (RI) da Literatura, método de revisão específico que sumariza a literatura teórica ou empírica anterior para prover o entendimento compreensivo de um fenômeno particular ou problema relacionado à saúde ${ }^{14}$.

Este método pode tornar os resultados de pesquisas mais acessíveis, reduzindo alguns obstáculos da utilização do conhecimento científico, pois possibilita ao leitor o acesso a diversas pesquisas realizadas, em um único estudo ${ }^{15}$.

Depois de estabelecida a questão norteadora, três bases de dados foram utilizadas como fonte de levantamento dos estudos: Literatura LatinoAmericana e do Caribe em Ciências da Saúde (Lilacs), Cumulative Index to Nursing and Allied Health Literature (CINAHL) e Medical Literature Analysis and Retrieval System on-line (Medline) e a combinação dos seguintes descritores: qualidade de vida (quality of life), idoso (aged and aged and over) e acidentes por quedas (accidental falls).

Os critérios de inclusão estabelecidos para a seleção dos artigos foram: artigos com resumos disponíveis relacionados à $\mathrm{QV}$ dos idosos $(\geq 60$ anos) que sofreram quedas, publicados nos idiomas português, espanhol e inglês, indexados nas bases de dados Lilacs, CINAHL e Medline, no período de janeiro de 1999 a outubro de 2009.

Os estudos foram examinados por um instrumento construído e validado ${ }^{16}$ que permitiu a identificação das publicações, sua caracterização quanto aos critérios de avaliação de QV e quanto à metodologia, considerando o delineamento de pesquisa dos artigos ${ }^{17-18}$ e o nível de evidência $^{19}$ dos mesmos.
O levantamento dos estudos foi realizado em outubro de 2009, concomitantemente nas três bases de dados. No cruzamento dos descritores, foi encontrado um total de 118 artigos, dos quais 15 se repetiam entre as bases de dados, restando 103 estudos em que, após serem lidos os resumos e aplicados os critérios de inclusão, foram selecionados 24 para a leitura na íntegra. Após a leitura destes artigos, sete foram selecionados, e, posteriormente, foram incluídos outros dois artigos referenciados nos estudos por atenderem aos critérios de inclusão, totalizando nove artigos que compuseram a amostra desta RI.

A análise dos artigos ocorreu de forma descritiva, de acordo com o instrumento de coleta de dados ${ }^{16}$, permitindo avaliar as seguintes características de cada artigo: identificação da publicação, critérios de avaliação de QV e metodologia dos estudos. Para a construção das categorias, utilizou-se a técnica de análise temática ${ }^{20}$.

\section{Resultados}

Foram analisados nove artigos na íntegra, e, após a análise de conteúdo temática, os temas abordados foram divididos nas seguintes categorias: Impacto da queda na QV (três artigos), Impacto do medo de queda na QV (três artigos), Programa de prevenção de quedas e QV (dois artigos), Relação entre fatores intrínsecos para risco de quedas e quedas na QV (um artigo).

Em relação à caracterização dos estudos, quanto ao ano de publicação, em 2008, 2004 e 2003 foram publicados dois artigos em cada ano e nos anos de 2007, 2005 e 2000 um artigo cada. Quanto à formação profissional do autor principal, um artigo foi publicado por médico, um por enfermeiro, dois por fisioterapeutas e em cinco não foi possível identificar a categoria profissional dos autores. De acordo com a instituição de origem dos autores principais, oito artigos estão vinculados a universidades e um está vinculado a uma Fundação.

No que se refere ao idioma, sete publicações foram em inglês e duas em português. Com relação ao país-sede do estudo, dois foram desenvolvidos no Canadá, dois em Taiwan, outros dois no Brasil, um nos Estados Unidos, um na Austrália e um na Holanda. Quanto ao periódico de publicação, foram detectados nove periódicos diferentes, sendo cinco revistas específicas de gerontologia e/ou geriatria.

Quanto aos critérios de avaliação de QV, em três artigos, os autores apresentaram uma defini- 
ção de QV e em seis não. Foram utilizados cinco instrumentos diferentes para mensurar a QV dos idosos nos estudos revisados (Quadro 1), sendo que em sete foi justificada a escolha dos mesmos e em dois não; quatro estudos referiram ter utilizado instrumentos validados para a população do estudo, e cinco não fizeram esta menção.

Os Quadros 2 e 3 apresentam as principais funções ou domínios afetados e a síntese do conhecimento, de acordo com as categorias temá- ticas dos estudos, os delineamentos de pesquisa e os níveis de evidência.

\section{Discussão}

Enquanto a expectativa de vida entre a população idosa tem aumentado devido às intervenções médicas e de saúde pública, estudos sobre a importância da QVRS nesta fase também têm

Quadro 1. Instrumentos de QV usados nos artigos analisados e as funções e itens de avaliação.

\begin{tabular}{|c|c|}
\hline Instrumentos & Funções e itens de avaliação \\
\hline $\begin{array}{l}\text { Instrumentos Genéricos: } \\
\text { EuroQol Instrument }{ }^{21} \\
\text { Medical Outcomes Study Short-Form } 12(\mathrm{SF}-12)^{9} \\
\text { Medical Outcomes Study Short-Form } 36(\mathrm{SF}-36)^{3,22,23} \\
\text { WHOQOL-bref }^{4,24,25}\end{array}$ & $\begin{array}{l}\text { Avaliam QV geral e as seguintes funções: física, mental ou } \\
\text { emocional, social, funcional ou de desempenho de papel }{ }^{27}\end{array}$ \\
\hline $\begin{array}{l}\text { Instrumentos Específicos: } \\
\text { Osteoporis Quality of Life questionnaire (OQLQ) }{ }^{26}\end{array}$ & $\begin{array}{l}\text { Avalia cinco domínios: atividades da vida diária, emoção, lazer, } \\
\text { funções física e } \text { social }^{26}\end{array}$ \\
\hline
\end{tabular}

Quadro 2. Síntese do conhecimento de acordo com as categorias temáticas: Impacto da Queda na QV e Impacto do Medo da Queda na QV.

\begin{tabular}{|c|c|c|c|c|}
\hline $\begin{array}{l}\text { Categoria } \\
\text { Temática }\end{array}$ & Estudos & $\begin{array}{l}\text { Delineamento/ } \\
\text { Nível de evidência }\end{array}$ & $\begin{array}{l}\text { Domínios de } \\
\text { QV afetados }\end{array}$ & Síntese do Conhecimento \\
\hline $\begin{array}{l}\text { Impacto da } \\
\text { Queda na QV }\end{array}$ & $\begin{array}{l}\text { Avaliaram o efeito } \\
\text { das quedas e suas } \\
\text { consequências na } \\
\text { QV dos idosos }{ }^{3,24-25}\end{array}$ & $\begin{array}{l}\text { Estudos descritivos - } \\
\text { nível } 6^{3,24,25}\end{array}$ & $\begin{array}{l}\text { Domínios psicológico/ } \\
\text { emocional e físico por } \\
\text { eles se sentirem/ } \\
\text { tornarem mais } \\
\text { dependentes, e } \\
\text { relacionado ao meio } \\
\text { ambiente, devido sua } \\
\text { relação com as } \\
\text { atividades do cotidiano. }\end{array}$ & $\begin{array}{l}\text { As quedas são frequentes nos } \\
\text { idosos e estes apresentaram escores } \\
\text { significativamente menores para } \\
\text { QV geral e para os domínios } \\
\text { psicológico/ emocional e físico em } \\
\text { relação aos que não apresentaram } \\
\text { quedas, mostrando que as quedas } \\
\text { resultam em consequências } \\
\text { negativas para a QV dessa } \\
\text { população. }\end{array}$ \\
\hline $\begin{array}{l}\text { Impacto do } \\
\text { Medo da } \\
\text { Queda na QV }\end{array}$ & $\begin{array}{l}\text { Avaliaram o impacto } \\
\text { do medo da queda } \\
\text { na QV da população } \\
\text { idosa e sua relação } \\
\text { com as quedas } \\
\text { apresentadas por esta } \\
\text { população }{ }^{9,22,23} \text {. }\end{array}$ & $\begin{array}{l}\text { Estudos descritivos - } \\
\text { nível } 6^{9,22} \text { Estudo } \\
\text { caso-controle - nível } \\
4^{23}\end{array}$ & $\begin{array}{l}\text { Grupo com alto medo } \\
\text { de quedas apresentou os } \\
\text { domínios físico, mental } \\
\text { e dor corporal } \\
\text { prejudicados. }\end{array}$ & $\begin{array}{l}\text { Os sujeitos que apresentaram } \\
\text { maior medo de quedas são aqueles } \\
\text { que apresentaram quedas } \\
\text { anteriores e piores escores, } \\
\text { estatisticamente significante, para } \\
\text { os domínios físico, mental e dor } \\
\text { corporal do que o grupo de idosos } \\
\text { com menor medo de quedas e do } \\
\text { que aqueles que não caíram } \\
\text { previamente. Este grupo também } \\
\text { tende a restringir suas atividades e } \\
\text { apresentam risco aumentado para } \\
\text { quedas futuras. }\end{array}$ \\
\hline
\end{tabular}


surgido $^{28}$. As reduções do número de quedas e dos danos causados por elas, por meio de programas de prevenção, podem contribuir para melhorar a $\mathrm{QV}^{10}$.

Embora as pesquisas tenham crescido nos últimos anos, os resultados do presente estudo mostraram que apenas dois artigos foram publicados no Brasil, em português; e nenhum artigo foi encontrado no idioma espanhol. Quanto à autoria principal, apenas um dos estudos foi realizado por enfermeiro, revelando a necessidade de estes profissionais investirem nesta área de pesquisa, na busca de subsídios para a assistência de enfermagem prestada aos idosos.

Com relação aos aspectos referentes à avaliação de QV, os resultados evidenciaram que somente três artigos utilizaram suas definições, esta deficiência foi observada em outros estudos de revisão ${ }^{10,29}$.

De acordo com o delineamento de pesquisa e o nível de evidência avaliado, houve predomínio de estudos descritivos, ou seja, seis artigos classificados com nível de evidência 6, considerado fra- co; um estudo caso-controle, nível 4; e dois Ensaios Clínicos Randomizados Controlados (ECRC), nível 2, de delineamento experimental, considerados evidências fortes; contudo, o nível 2 de evidência na pesquisa em enfermagem ainda é restrito.

Quanto às categorias temáticas apresentadas no Quadro 2, na categoria Impacto da queda na $\mathrm{QV}$, as quedas foram frequentes nos idosos e ocasionaram consequências negativas para sua QV. O estudo ${ }^{28}$ que avaliou a QV em idosos com fratura de quadril devido a quedas corroborou que estas interferem na QV dessa população e que planos de intervenção para alta hospitalar podem diminuir o tempo de internação, algumas consequências pós-alta hospitalar, a razão de readmissão hospitalar, a razão de sobrevivência, além de possibilitar melhora no desempenho das atividades da vida diária e melhores condições de QV para os mesmos.

Com relação à categoria Impacto do medo da queda na QV, foi evidenciado que os idosos com maior medo de quedas foram os que mais

Quadro 3. Síntese do conhecimento de acordo com as categorias temáticas: Programa de Prevenção de Quedas e QV e Relação entre Fatores Intrínsecos para Risco de Quedas e Quedas na QV.

\begin{tabular}{|c|c|c|c|c|}
\hline $\begin{array}{l}\text { Categoria } \\
\text { Temática }\end{array}$ & Estudos & $\begin{array}{l}\text { Delineamento/ } \\
\text { Nível de evidência }\end{array}$ & $\begin{array}{l}\text { Domínios de } \\
\text { QV afetados }\end{array}$ & Síntese do Conhecimento \\
\hline $\begin{array}{l}\text { Programa de } \\
\text { Prevenção de } \\
\text { Quedas e QV }\end{array}$ & $\begin{array}{l}\text { Comparou programas } \\
\text { de prevenção de } \\
\text { quedas: Educação, } \\
\text { Exercícios e Avaliação e } \\
\text { Modificação } \\
\text { Domiciliar }{ }^{30} \text { e avaliou o } \\
\text { custo-eficácia do }_{\text {programa de Prevenção }} \\
\text { Multidisciplinar (com } \\
\text { recomendações para } \\
\text { mudanças } \\
\text { comportamentais de } \\
\text { ambiente) com } \\
\text { Cuidado de Saúde } \\
\text { Usual }{ }^{21} \text { em idosos sobre } \\
\text { a QV dessa população. }\end{array}$ & $\begin{array}{l}\text { Ensaio Clínico } \\
\text { Randomizado } \\
\text { Controlado (ECRC) - } \\
\text { nível } 2^{21,30}\end{array}$ & $\begin{array}{l}\text { Os idosos que } \\
\text { relataram quedas } \\
\text { apresentaram } \\
\text { piores escores } \\
\text { para todos os } \\
\text { domínios de QV. }\end{array}$ & $\begin{array}{l}\text { A QV dos idosos dos programas de } \\
\text { prevenção de quedas aumentou } \\
\text { durante o seguimento nos dois } \\
\text { estudos. Nos idosos que } \\
\text { apresentaram quedas, o grupo que } \\
\text { realizou Exercícios apresentou } \\
\text { melhores resultados nos escores de } \\
\text { QV do que as outras duas } \\
\text { intervenções. O Grupo de Programa } \\
\text { Multidisciplinar preveniu mais } \\
\text { quedas do que o seu Grupo Controle } \\
\text { (GC), porém o GC teve melhores } \\
\text { escores para QV geral e não houve } \\
\text { diferença estatisticamente } \\
\text { significante para custos e eficácia } \\
\text { entre os grupos. }\end{array}$ \\
\hline $\begin{array}{l}\text { Relação entre } \\
\text { Fatores } \\
\text { Intrínsecos } \\
\text { para Risco de } \\
\text { Quedas e } \\
\text { Quedas na } \\
\text { QV }\end{array}$ & $\begin{array}{l}\text { Examinaram a relação } \\
\text { entre queda e QV em } \\
\text { mulheres idosas com } \\
\text { osteoporose }^{26} \text {. }\end{array}$ & $\begin{array}{l}\text { Estudo descritivo - } \\
\text { nível } 6^{26}\end{array}$ & $\begin{array}{l}\text { Domínio } \\
\text { Emocional }\end{array}$ & $\begin{array}{l}\text { O aumento da cifose e o Estado } \\
\text { Emocional (medo de queda) são } 2 \\
\text { fatores intrínsecos associados com } \\
\text { quedas recentes em idosas com } \\
\text { osteoporose.As idosas que } \\
\text { apresentaram melhor estado } \\
\text { emocional e menos medo de quedas } \\
\text { foram as que tiveram menor quedas } \\
\text { recentes. }\end{array}$ \\
\hline
\end{tabular}


as sofreram e apresentaram piores escores para QV e assim, como nesta revisão, estudos ${ }^{7,31}$ mostraram que idosos que sofreram quedas anteriores relataram mais medo de sofrê-las do que os que não caíram, e o estudo ${ }^{31}$ encontrou entre as mulheres na faixa etária de 60 a 69 anos maior medo de cair, aquelas que apresentaram de moderado a alto medo de cair relataram piores escores para funções física, social, funcional, dor corporal, vitalidade e saúde geral, e os homens com moderado grau de medo de cair apresentaram piores escores para as funções social e funcional e para saúde geral. Nesta revisão, os idosos com alto medo de cair relataram piores escores nas funções física e mental e na dor corporal.

Quanto à categoria Programa de prevenção de quedas e $\mathrm{QV}$, os grupos de idosos que tiveram quedas e realizaram exercícios apresentaram melhores resultados para QV do que os grupos que caíram e receberam orientações para prevenção de quedas, como modificações comportamentais em relação ao ambiente, e educacionais. Uma Revisão Sistemática (RS) ${ }^{10}$ avaliou Ensaios Clínicos Randomizados Controlados (ECRC) que envolveram intervenções para prevenção de quedas, como uma avaliação dos efeitos da QV dos idosos, e encontrou poucos estudos que usaram a QV com este objetivo, seis em 12 intervenções obtiveram resultados positivos e, destes, oito reportaram resultados relacionados às quedas, mas nem todos compararam a QV entre os que sofreram e os que não sofreram quedas. Nos dois estudos desta RI, um destes ${ }^{30}$ também está incluso na RS, eles realizaram esta comparação e encontraram a QV prejudicada para os idosos que sofreram quedas, mas com o decorrer do programa de intervenção para prevenção de quedas, a QV melhorou, sendo considerados resultados positivos.

Com relação à categoria Relação entre fatores intrínsecos para risco de quedas e quedas na $\mathrm{QV}$, as idosas com osteoporose que sofreram quedas apresentaram a função emocional prejudicada. A osteoporose também foi comum em mulheres no estudo ${ }^{32}$, cuja avaliação de um programa de reabilitação para idosos com fratura de quadril evidenciou a função física prejudicada e o medo de cair. A osteoporose também foi predominante no sexo feminino, e esta fragilidade das mulheres idosas fez com que tivessem maior incidência de medo de cair quando comparadas com os homens ${ }^{31}$. A queda em idosos com idade acima de 85 anos foi preditiva para pior saúde física, emoções negativas e menos atividade física do que entre idosos com idade inferior a esta ${ }^{8}$.
Ressaltam-se, como restrições deste estudo, a delimitação dos idiomas português, espanhol e inglês, a utilização de apenas três bases de dados e a não inclusão de dissertações e teses, o que pode ter excluído estudos considerados importantes.

\section{Conclusão}

Este estudo possibilitou caracterizar a produção científica quanto aos aspectos metodológicos dos estudos sobre QV apresentada pelo idoso que sofreu queda. No total, nove artigos preencheram os critérios de inclusão e fizeram parte desta RI.

Com relação à categorização dos estudos, como lacunas no conhecimento, observou-se a escassez de estudos de intervenção que retratassem evidências fortes, pois a maioria dos encontrados foi descritiva, classificada como nível de evidência 6, considerada fraca. Detectou-se, também, baixa produção científica tanto no Brasil quanto na América Latina, sendo apenas dois estudos publicados no Brasil, em português; e com a participação do enfermeiro em apenas um estudo como autor principal, fazendo-se necessária uma reflexão sobre a temática, para que novas pesquisas sejam desenvolvidas e divulgadas.

Quanto aos aspectos relacionados à $\mathrm{QV}$, apenas três estudos a definiram, quatro não mencionaram se utilizaram instrumentos validados para sua população e dois não justificaram suas escolhas; é importante a utilização de instrumentos validados para a confiabilidade dos resultados.

Quatro categorias temáticas foram estabelecidas, após a análise dos estudos: Impacto da queda na QV, Impacto do medo da queda na $\mathrm{QV}$, Programa de prevenção de quedas e QV e Relação entre fatores intrínsecos para risco de queda e queda na QV. E observou-se que: as quedas são frequentes nos idosos, sendo a maioria devido aos fatores intrínsecos; e aqueles idosos que já caíram têm mais medo e maior chance de cair novamente, eles apresentaram déficits em funções ou domínios da QV, tais como: funções física, emocional ou mental, dor corporal e relacionados ao meio ambiente. Sendo que programas de intervenção de quedas podem melhorar sua $\mathrm{QV}$ após a queda.

Portanto, de acordo com os resultados desta RI, sugere-se aos profissionais de saúde, inclusive aos enfermeiros, maior atuação tanto na assistência quanto na produção de pesquisas direcionadas aos idosos, desenvolvendo estudos de intervenção para prevenção de quedas que pos- 
sam ser aplicados na prática clínica e que possibilitem melhorar a QV dos idosos.

\section{Colaboradores}

AC Nicolussi participou da elaboração, análise dos dados, redação e análise crítica do artigo; JRS Fhon e CAV Santos participaram da elaboração, análise dos dados e redação do artigo; L Kusumota, S Marques e RAP Rodrigues participaram da revisão crítica do artigo.

\section{Referências}

1. Carvalho Filho ET, Papaleo Netto M. Geriatria: fundamentos, clínica e terapêutica. 2a. ed. São Paulo: Atheneu; 2006.

2. Paschoal SMP, Franco RP, Salles RFN. Epidemiologia do Envelhecimento In: Netto MP, organizador. Tratado de Gerontologia. São Paulo: Editora Atheneu; 2007. p. 39-56.

3. Santiago ALM, Moreira JS, da Silva ÉG, Fernandes VL, Dias RC, Dias JMD. Mobility, falls and quality of life in dwelling elderly [Portuguese]. Fisioterapia em Movimento 2004; 17(2):29-36.

4. Gama Z, Gomez-Conesa A. Factores de riesgos de caídas em ancianos: revisión sistemática. Rev Saude Publica 2008; 42(5):946-956.

5. Schiaveto FV. Avaliação do risco de quedas em idosos na comunidade [dissertação]. Ribeirão Preto (SP): Universidade de São Paulo; 2008.

6. Aguiar CF, Assis M. Perfil de mulheres idosas segundo a ocorrência de quedas: estudo de demanda no Núcleo de Atenção ao idoso da UNATI / UERJ. Rev Bras Geriatr Gerontolol 2009; 12(3):391-404.

7. Miller WC, Deathe AB, Speechley M, Koval J. The influence of falling, fear of falling, and balance confidence on prosthetic mobility and social activity among individuals with a lower extremity amputation. Arch Phys Med Rehabil 2001; 82(9):1238-1244.

8. Ruthig JC, Chipperfield JG, Newall NE, Perry RP, Hall NC. Detrimental effects of falling on health and well-being in later life: the mediating roles of perceived control and optimism. J Health Psychol 2007; 12(2):231-248.

9. Li F, Fisher KJ, Harmer P, McAuley E, Wilson NL. Fear of falling in elderly persons: association with falls, functional ability, and quality of life. J Gerontology B Psychol Sci Soc Sci 2003; 58(5):P283-90.

10. Vaapio SS, Salminen MJ, Ojanlatva A, Kivelä SL. Quality of life as an outcome of fall prevention interventions among the aged: a systematic review. Euro J Public Health 2009; 19(1):7-15.

11. The WHOQOL Group. The World Health Organization quality of life assessment (WHOQOL): position paper from the World Health Organization. Soc Sci Med 1995; 41(10):1403-1409.

12. Bowling A. Measuring Disease. $2^{\text {nd }}$ edition. Philadelphia USA: Open University Press; 2001.

13. Vecchia RD, Ruiz T, Bocchi SCM, Corrente JE. Qualidade de Vida na Terceira Idade. Rev Bras Epidemiol 2005; 8(3):246-252.

14. Broome ME. Integrative literature reviews for the development of concepts. In: Rodgers BL, Knalf $\mathrm{KA}$, editors. Concept development in nursing: foundations, techniques and applications. Philadelphia: Sounders Company; 2000. p. 231-250. 
15. Whittemore $\mathrm{R}$, Knalf $\mathrm{K}$. The integrative review: updated methodology. J Ad Nursing 2005; 52(5):546553.

16. Nicolussi AC. Qualidade de vida de pacientes com câncer de cólon e reto: revisão integrativa da literatura [dissertação]. Ribeirão Preto (SP): Universidade de São Paulo; 2008.

17. Polit DF, Bech CT, Hungler BP. Fundamentos de pesquisa em enfermagem. $5^{a}$ ed. Porto Alegre: ArtMed Editora; 2004.

18. Lobiondo-Wood G, Haber J. Nursing Research: methods and critical appraisal for evidence-based practice. $6^{\text {th }}$. St. Louis: Mosby/ Elsevier, 2006.

19. Melnik BM, Fineout-Overholt E. Making the case for evidence-based practice. In: Melnik BM, FineoutOverholt E, editors. Evidence-based practice in nursing \& healthcare. A guide to best practice. Philadelphia: Lippincott Williams Wilkins; 2005. p. 3-24.

20. Minayo MCS. O desafio do conhecimento: pesquisa qualitativa em saúde. $8^{a}$ ed. São Paulo: Hucitec; 2006.

21. Hendriks MR, Evers SM, Bleijlevens MH, van Haastregt JC, Crebolder HF, van Eijk JT. Cost-effectiveness of a multidisciplinary fall prevention program in community-dwelling elderly people: a randomized controlled trial. Int J Technol Assess Health Care 2008; 24(2):193-202.

22. Cumming RG, Salkeld G, Thomas M, Szonyi G. Prospective study of the impact of fear of falling on activities of daily living, SF-36 scores, and nursing home admission. J Gerontol Med Sci 2000; 55A(5): M299-305.

23. Brouwer B, Musselman K, Culham E. Physical function and health status among seniors with and without a fear of falling. Gerontology 2004; 50(3):135141.

24. Ribeiro AP, Souza ET, Atie S, Souza AC, Shilithz AO. A Influência das quedas na qualidade de vida de idosos. Cien Saude Colet 2008; 13(4):1265-1273.

25. Hwang H, Liang W, Chiu Y, Lin M. Suitability of the WHOQOL-BREF for community-dwelling older people in Taiwan. Age and Ageing 2003; 32(6):593-600.
26. Arnold CM, Busch AJ, Schachter CL, Harrison L, Olszynski W. The relationship of intrinsic fall risk factors to a recent history of falling in older women with osteoporosis. J Orthop Sports Phys Ther 2005; 35(7):452-460.

27. Fayers PM, Machin D. Quality of life. The assessment, analysis and interpretation of patient-reported outcomes. $2^{\text {nd }}$ ed. New Jersey: John Wiley \& Sons Ltd; 2007.

28. Huang T, Liang S. A randomized clinical trial of the effectiveness of a discharge planning intervention in hospitalized elders with hip fracture due to falling. J Clin Nurs 2005; 14(10):1193-1201.

29. Nicolussi AC, Sawada NO. Fatores que influenciam a qualidade de vida de pacientes com câncer de cólon e reto. Acta Paul Enferm 2010; 23(1):125-130.

30. Lin M, Wolf SL, Hwang H, Gong S, Chen C. A randomized, controlled trial of fall prevention programs and quality of life in older fallers. J Am Geriatr Soc 2007; 55(4):499-506.

31. Suzuki M, Ohyama N, Yamada K, Kanamori M. The relationship between fear of falling, activities of daily living and quality of life among elderly individuals. Nursing Health Sciences 2002; 4(4):155-161.

32. Petrella RJ, Payne M, Myers A, Overend T, Chesworth B. Physical function and fear of falling after hip fracture rehabilitation in the elderly. Am J Phys Med Rehabil 2000; 79(2):154-160.

Artigo apresentado em 16/06/2010

Aprovado em 28/09/2010

Versão final apresentada em 06/10/2010 